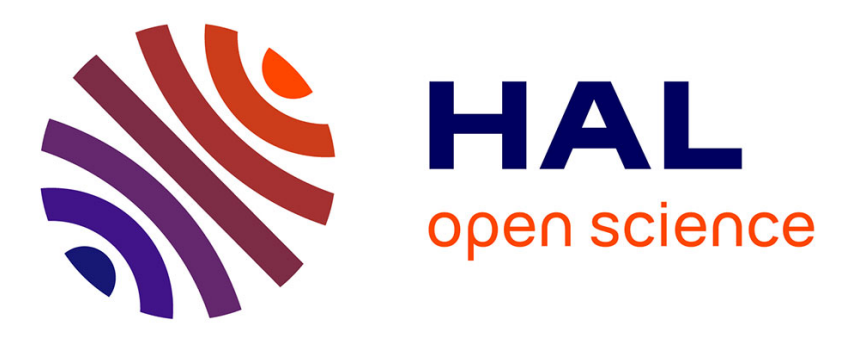

\title{
The evolution with strain of the stored energy in different texture components of cold-rolled if steel revealed by high resolution X-ray diffraction
}

Aurélie Wauthier-Monnin, T Chauveau, Olivier Castelnau, Hélène Réglé,

Brigitte Bacroix

\section{To cite this version:}

Aurélie Wauthier-Monnin, T Chauveau, Olivier Castelnau, Hélène Réglé, Brigitte Bacroix. The evolution with strain of the stored energy in different texture components of cold-rolled if steel revealed by high resolution X-ray diffraction. Materials Characterization, 2015, 104, pp.31-41. 10.1016/j.matchar.2015.04.005 . hal-02137003

\section{HAL Id: hal-02137003 \\ https://hal.science/hal-02137003}

Submitted on 22 May 2019

HAL is a multi-disciplinary open access archive for the deposit and dissemination of scientific research documents, whether they are published or not. The documents may come from teaching and research institutions in France or abroad, or from public or private research centers.
L'archive ouverte pluridisciplinaire HAL, est destinée au dépôt et à la diffusion de documents scientifiques de niveau recherche, publiés ou non, émanant des établissements d'enseignement et de recherche français ou étrangers, des laboratoires publics ou privés. 


\title{
The evolution with strain of the stored energy in different texture components of cold-rolled IF steel revealed by high resolution $\mathrm{X}$-ray diffraction
}

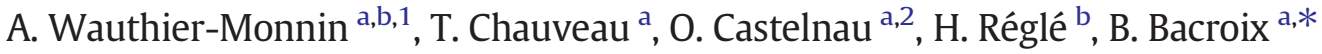 \\ a LSPM-CNRS, Université Paris 13, 99, Av. J.B. Clément, 93430 Villetaneuse, France \\ b ArcelorMittal Research Voie Romaine BP 30320, 57283 Maizières-les Metz, France
}

Keywords:

IF steels

Stored energy

X-ray diffraction

Dislocation densities

\begin{abstract}
A B S T R A C T
During the deformation of low carbon steel by cold-rolling, dislocations are created and stored in grains depending on local crystallographic orientation, deformation, and deformation gradient. Orientation dependent dislocation densities have been estimated from the broadening of X-ray diffraction lines measured on a synchrotron beamline. Different cold-rolling levels (from 30\% to 95\% thickness reduction) have been considered. It is shown that the present measurements are consistent with the hypothesis of the sole consideration of screw dislocations for the analysis of the data. The presented evolutions show that the dislocation density first increases within the $\alpha$ fiber $(=\{\mathrm{hkl}\}<110>$ ) and then within the $\gamma$ fiber $(=\{111\}<\mathrm{uvw}>$ ). A comparison with EBSD measurements is done and confirms that the storage of dislocations during the deformation process is orientation dependent and that this dependence is correlated to the cold-rolling level. If we assume that this dislocation density acts as a driving force during recrystallization, these observations can explain the fact that the recrystallization mechanisms are generally different after moderate or large strains.
\end{abstract}

\section{Introduction}

Polycrystalline metals, when subjected to a heat treatment after cold deformation, usually undergo a drastic microstructural transformation by the so-called static recrystallization process, during which new and dislocation-free grains are formed within the deformed microstructure [1]. This induces in the material a strong modification of the crystallographic texture and in turn of the physical and mechanical properties. This texture evolution is accompanied by a release of the energy stored in the material during plastic deformation, which is known to act as a driving force for nucleation and/or growth of new grains [1]. It is thus important, in order to understand, model and control the texture evolution during annealing to be able to quantitatively characterize this stored energy, and especially to be able to assess its evolution with strain and its dependence with crystallographic orientation [2].

Indeed, it is quite common to relate this stored energy to the production and organization of dislocations during deformation. If some basic linear relationships between stored energy and dislocation density can thus be found in the literature [3-5] (see below), the way

\footnotetext{
* Corresponding author.

E-mail address: brigitte.bacroix@univ-paris13.fr (B. Bacroix).

${ }^{1}$ Now at Sorbonne universités, Université de technologie de Compiègne, CNRS, Laboratoire Roberval, CS 60319, 60200 Compiègne, France.

2 Now at PIMM, CNRS, Arts et Métiers ParisTech, 151 bd de L'hôpital, 75013 Paris, France.
}

these dislocations are locally organized is also well documented and shown to strongly depend on the orientation of the grain. A number of papers relate e.g. experimental data obtained through Transmission Electron Microscopy (TEM) [5-7] or Electron Back Scattering Diffraction within a Scanning Electron Microscope (SEM/EBSD) [8-10], which allow their authors to distinguish various types of dislocation arrangements as a function of strain, strain mode, material and grain orientation. In all investigated polycrystalline metals deformed up to large strains at room temperature, it is generally recognized that a high proportion of stored dislocations are organized into "patterns" within individual grains, composed of elements such as dislocation cells or walls. Simultaneously, some more isolated dislocations are also observed, which are either randomly distributed inside the cells (between the dislocation walls), or more localized close to the grain boundaries to accommodate the strain incompatibilities between neighboring grains. And classically, the total dislocation density into one grain $\rho_{\text {tot }}$ can thus be decomposed as $[4,11]$

$\rho_{\text {tot }}=\rho_{w}+\rho_{c}+\rho_{G B}$

where $\rho_{w}$ is the density of the dislocations constituting the cell walls, $\rho_{c}$ the density of the dislocations randomly distributed within the cells and $\rho_{G B}$ the density of the dislocations present at grain boundaries. As the dislocations at grain boundaries as well as the one composing the cell walls involve misorientations, both can be grouped under the classical expression of geometrically necessary dislocations 
(GNDs) [12,13], whereas the randomly distributed dislocations are called statistically stored dislocations (SSD). Eq. (1a) can then be rewritten more simply as

$\rho_{\text {tot }}=\rho_{G N D}+\rho_{S S D}$

In order to measure these various dislocation densities, TEM, SEM/EBSD and X-ray Diffraction (XRD) are classically used. However, these techniques do not necessarily allow to distinguish all various dislocation types and thus to measure all terms in Eq. (1b). With TEM or SEM/EBSD, $\rho_{w}$ or $\rho_{G B}$ is easily deduced from local misorientation measurements. These measurements are more rarely completed by the assessment of $\rho_{c}$, which involves tedious and long counting procedures [4,6]. With XRD, which is a more statistical method, the analysis of the shape of a Bragg peak (and especially the broadening of the peak) measured inside a grain or a texture component is used to assess one "average" dislocation density, usually estimated to be the sole density of the isolated dislocations $\rho_{c}[11,14-16]$. The main argument advanced to eliminate any influence of the dislocation boundaries observed in highly deformed materials on peak broadening is of different types. It is most often claimed that essentially statistical dislocations (SSD) can affect peak broadening: this is for example an implicit assumption of Wilkens model [17]. More rarely, it is argued that the density $\rho_{c}$ is by far the greatest (by a factor of 10) and that the other two can thus be neglected [11]. This last argument was advanced for measurements performed in steel. However, most of TEM observations performed on $\mathrm{Al}$ [6], $\mathrm{Cu}$ [4] or steels [5] do find the opposite result, i.e. show that $\rho_{w} / \rho_{c}$ varies between 3 and 10 (instead of $1 / 10$ in [11]). Additionally, some recent work aiming at comparing both SEM and X-ray methods conclude to a final ratio $\rho_{w} / \rho_{c}$ of 1 [18].

Once the various dislocation densities have been evaluated, several equations are used to derive the associated stored energy; for example, if only GNDs are considered, the associated stored energy is usually expressed as [4]

$E_{s}=\frac{\mu b^{2}}{2} \rho_{G N D}$

where $\rho_{G N D}$ is calculated from the local misorientation measured by TEM or EBSD through a linear relationship. In Eq. (2), $\mu$ is the shear modulus and $b$ the norm of the Burgers vector of the dislocations. As for measurements performed by X-ray diffraction, the expression used to evaluate the stored energy is in this case [19]

$E_{S}=\frac{\alpha \mu b^{2}}{2} \rho_{X R D} \ln \frac{R_{e}}{b}$

where $\rho_{X R D}$ and $R_{e}$ are derived from the peaks measured by XRD (see below). This expression has been shown by Wilkens $[17,19]$ to be valid, not only for randomly distributed dislocations, but also for what Wilkens calls restrictedly random distributions of dislocations, which can be seen in a way as random distributions by parts. In Eq. (3), there is also an extra parameter $\alpha$ which depends on the character (edge, screw or mixed) of the considered dislocations (see Section 3).

For steels, the first XRD measurements performed after rolling by Every and Hatherley [14] established the following hierarchy for the stored energy $\left(E_{s}\right)$ as a function of fiber orientations $\{\mathrm{hkl}\}^{3}$

$E_{s}\{200\}<E_{s}\{211\}<E_{s}\{222\}<E_{s}\{110\}$.

These authors explicitly mention that, to their opinion, only the randomly distributed dislocations are taken into consideration in

\footnotetext{
${ }^{3}$ Here $\{$ hkl $\}$ represents the ensemble of orientations having a $\{\mathrm{hkl}\}$ plane parallel to the rolling plane. See Section 2 for more details about the definitions of orientations and orientation fibers.
}

their measurements. This hierarchy was since then never contradicted by more recent measurements, but the published quantitative values are relatively scattered: Borbely et al. [11] estimate the ratio $E_{s}$ $\{222\} / E_{s}\{200\}$ to 2.8 after $48 \%$ rolling strain on UHP (ultra high purity) iron and to 3.6 after $88 \%$ rolling whereas a factor of 1.8 is reported by Castelnau et al. [20] for an IF (Interstitial Free) steel after $90 \%$ rolling. Using neutron diffraction and a quite different method for deriving the stored energy directly from the diffraction peaks, Rajmohan et al. [21] also found a factor close to 2 for this ratio after $80 \%$ rolling. It is worth mentioning though that, apart for differences in the used X-ray or neutrons devices or in the investigated steels, the methods used for the instrumental correction and analysis of the data may differ strongly from one publication to another.

Thus, the aims of the present paper are: (i) to present some recent data concerning the quantitative assessment of dislocation densities by XRD as a function of orientation and strain in a low carbon steel deformed in rolling, (ii) to discuss in some details the link between the obtained values and the various dislocation densities introduced above and to propose an analysis method for XRD data, (iii) to validate this method by some complementary EBSD measurements and (iv) based on all these experimental data, to propose a general scenario of microstructural evolution during rolling as a function of strain and grain orientation and to show that it is already consistent with some published data concerning nucleation and growth of new grains during annealing of low carbon steels, after various amounts of strain.

The present paper is thus organized as follows: the material, the used experimental techniques and the characterization of the crystallographic texture are first described in Section 2, the method used for the analysis of the XRD data is then detailed and justified in Section 3, the data obtained after various deformation levels, as well as some complementary EBSD measurements are then presented in Section 4; and the comparison of all these data allows us to discuss the validity of our approach before drawing some conclusions in Section 5.

\section{Material and experimental procedures}

\subsection{Material and thermomechanical processing}

The investigated material is an Interstitial Free Steel (IF steel), i.e. a low carbon steel often used in industry for automotive applications. The alloying elements present in the material are given in Table 1: in the present case, all interstitial dissolved nitrogen and carbon atoms are captured by titanium mainly. The deep-drawing properties of this steel depend on its final crystallographic texture, resulting from the overall thermomechanical processing route, composed of a first hot rolling step, followed by an intense cold rolling step and a subsequent controlled heat treatment designed to achieve complete recrystallization (not studied in the present paper, but during which the basic nucleation and growth mechanisms will be directly affected by the distribution of stored energy which is studied here). In the present case, the selected IF steel has reached a final thickness of $3 \mathrm{~mm}$ and an average grain size of $20 \mu \mathrm{m}$ after hot rolling. It has then been deformed by cold-rolling in several steps, in a laboratory rolling mill up to a final reduction of 93\%, which corresponds to a final thickness of $0.23 \mathrm{~mm}$.

\subsection{Microstructure and texture examination after cold deformation}

The microstructures of the material have been analyzed after various rolling strains (between 15\% and 93\%) by Electron Back Scattering

Table 1

Chemical composition of the investigated IF steel: concentration of the alloying elements in $10^{-3}$ wt.\%.

\begin{tabular}{lllllllll}
\hline $\mathrm{C}$ & $\mathrm{Mn}$ & $\mathrm{S}$ & $\mathrm{Al}$ & $\mathrm{N}$ & $\mathrm{P}$ & $\mathrm{Cu}$ & $\mathrm{Ti}$ & $\mathrm{Nb}$ \\
\hline 2.8 & 154.2 & 7.8 & 31 & 2.2 & 14.5 & 6.3 & 49.1 & 2.8 \\
\hline
\end{tabular}


Diffraction (EBSD) on a Cambridge S360 scanning electron microscope equipped with the automatic Orientation Imaging Microscopy (OIM) software from Tex-SEM laboratories Inc. for data acquisition. The HKL software from Channel 5 has also been used for part of the data analysis. For EBSD observations, the samples were mechanically polished with silicon carbide paper down to grade 4000, then electro-polished in the Struers A2 solution (composed of perchloric acid and butoxyethanol).

The crystallographic textures of these samples have been measured in laboratory by XRD on an Inel four circle diffractometer in Bragg Brentano geometry. The main purpose of these measurements is to be able to select the diffracting positions for the subsequent high resolution X-ray measurements (Section 2.3). From the three $\{110\},\{200\}$ and $\{211\}$ experimentally determined pole figures, the Orientation Distribution Function (ODF) has then been calculated with the Labotex ${ }^{\mathbb{O}}$ Software, which is based on the ADC (Arbitrarily Defined Cells) iterative discrete method, which allows to describe the texture as a discrete set of orientations and associated volume fractions [22]. For the result analysis and for the selection of the orientations to be studied in high resolution X-Ray diffraction, we restricted ourselves to the main orientations which are usually found after rolling and annealing, and which are all located within the $\varphi_{2}=45^{\circ}$ section of the Euler space $^{4}$ (see Fig. 1). Orientations A, B and C are located on the so-called $\alpha$ fiber $=\{\mathrm{hkl}\}<110>,{ }^{5}$ designating the group of orientations presenting $\mathrm{a}<110>$ direction parallel to the rolling direction. Orientations $\mathrm{C}$ and $\mathrm{F}$ are located on the $\gamma$ fiber $=\{111\}<\mathrm{uvw}>$ designating the group of orientations having a $\{111\}$ plane parallel to the rolling plane. The orientations $\mathrm{C}^{\prime}$ and $\mathrm{F}^{\prime}$ are symmetrically equivalent orientations of $\mathrm{C}$ and $\mathrm{F}$ respectively which are statistically as equally present as $\mathrm{C}$ and $\mathrm{F}$, because of the orthotropic symmetry of the rolling process. The orientations $\mathrm{D}$ and $\mathrm{E}$ (which are sometimes present after very large strains and may thus play a role during recrystallization) are slightly shifted from the $\gamma$ fiber (from 6 and $11^{\circ}$ respectively).

The texture evolution during cold rolling is presented in Fig. 2. In the initial state, which corresponds to hot rolling, the texture is quite scattered as a result of a complex process involving hot deformation, recrystallization and phase transformation. Then, during cold deformation, a classical rolling texture is formed, which consists in a partial $\alpha$ fiber ranging from $\{001\}<110>$ towards $\{111\}<110>$ and a complete $\gamma$ fiber. It is seen in this figure that the exact position of the absolute maximum in this section evolves with strain and seems to be close to the $\mathrm{E}$ orientation, intermediate between the $\mathrm{B}$ and $\mathrm{F}$ orientations, after large strains. To be more precise, the percentages of the main orientations have been calculated from the ODFs (with a spread of $\pm 7.5^{\circ}$, classically selected for moderate to high values of strain, as in the present work). Results are reported in Table 2 and graphically presented in Fig. 3 as a function of strain. It is clear from the figure, that the B and $E$ orientations become the strongest after $60 \%$ strain, to the expense of the A orientation. The $\gamma$ fiber ( $\mathrm{C}$ and F orientations) is only slightly reinforced during rolling (up to $75 \%$ rolling), whereas the D component remains at the lowest level, very close to that of the neighboring $\mathrm{F}$ orientation. However, it is important to note that, due to the selected spread, the orientations F and D may significantly overlap.

\subsection{Acquisition of high resolution diffraction data}

\subsubsection{Experimental setup}

As the analysis of the XRD data for stored energy is based on peak broadening, it is important first to eliminate any influence of the instrumental device on it. In the present study, high resolution data were

\footnotetext{
${ }^{4}$ The Euler space is the commonly used orientation space in which orientation distribution functions can be represented and in which each orientation is represented by 3 Euler angles $\left(\varphi_{1}, \Phi, \varphi_{2}\right)$ which vary between $0^{\circ}$ and $90^{\circ}$ because of the orthotropic symmetry.

${ }^{5}$ The metallurgical notation in terms of Miller indices $\{\mathrm{hkl}\}<\mathrm{uvw}>$ is used throughout this paper to describe orientations and orientations fibers; $\{\mathrm{hkl}\}$ refers to the Miller indices of the rolling plane and $<\mathrm{uvw}>$ are the ones of the rolling direction.
}

$$
\begin{aligned}
& A=\{100\}<110> \\
& B=\{112\}<110> \\
& C=\{111\}<110> \\
& F=\{111\}<112> \\
& D=\{554\}<225> \\
& E=\{223\}<582>
\end{aligned}
$$

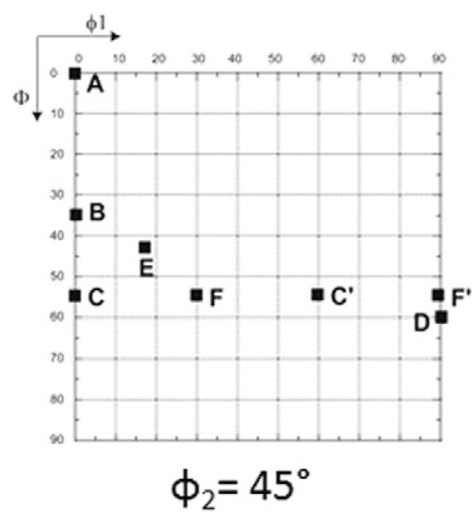

(a)

(b)

Fig. 1. (a) Selected orientations for XRD measurements and (b) their representation in the Euler section at $\varphi_{2}=45^{\circ}$.

obtained at BM02 beamline (7-circles diffractometer of the Synchrotron ESRF, Grenoble, France) at an energy E of $20 \mathrm{keV}$, thanks to (i) the very small beam divergence and bandwidth $\left(\Delta E / E\right.$ about $\left.10^{-4}\right)$, (ii) the use of an analyzer crystal in the diffracted beam, and (iii) a solid state detector for photon counting. It is worth mentioning that such a resolution cannot be reached using laboratory goniometers unless the acquisition time becomes excessively large. With this energy, the attenuation length is $\sim 50 \mu \mathrm{m}$ for iron, which is thus much larger than the grain size (which is about $15 \mu \mathrm{m}$ ) even for the less deformed sample [10]. Moreover, the beam cross section was selected to be $3 \mathrm{~mm}^{2}$ in order to scan a large number of grains simultaneously. It is a difficult task to evaluate the exact number of grains that have been scanned simultaneously, but we estimate that it must be at least several thousand for each specific orientation for all specimens; this guarantees our data to be statistically representative for the texture component as a whole. Additionally, the diffracting volume varies with the volume fraction of the scanned orientation; this feature may have an influence of the profiles, especially in terms of overlapping of various orientations. For each measured $\theta-2 \theta$ line profile, 310 data points were acquired (each at different $\theta$ values), with $\sim 10$ points describing the peak itself and $\sim 300$ points describing the intensity decay in the peak tails, important for line profile analysis. This discretization was selected such that the complete measurement scan always corresponds to 30 times the full width at half maximum (FWHM) of the profile. As a result, the whole amplitude of the $2 \theta$ scans was equal to $0.9^{\circ}$ for the less deformed specimens but increased to $2.9^{\circ}$ for the most deformed ones due to the broadening of the Bragg peaks with strain.

A reference powder of $\mathrm{CeO}_{2}$ (Reference NIST SRM674a) was first used to characterize the resolution of the setup at BM02 (so-called Caglioti curve), and an almost constant and low value $\left(\sim 0.03^{\circ}\right)$ of the Integral Breadth (IB) of the peak has been recorded in the investigated $2 \theta$ range (see Fig. 4), as typically found for synchrotron setups. The IB measured on two deformed samples is also provided for comparison, showing that, for highly deformed specimens, peak broadening is mostly due to the lattice distortion by dislocations, and that the instrumental broadening coming from the optical aberrations can be neglected in our analysis. In fact, the treatment of the peaks has been performed with and without subtracting this instrumental broadening in the worst cases, and it was concluded that this correction has only little influence on the final data.

\subsubsection{Typical high resolution $X R D$ data}

The experimental set-up parameters were then selected to analyze the six specific crystallographic orientations indicated in Fig. 1 separately, i.e. to minimize a possible overlap between several of the investigated orientations (this is hardly possible though for the F and D 


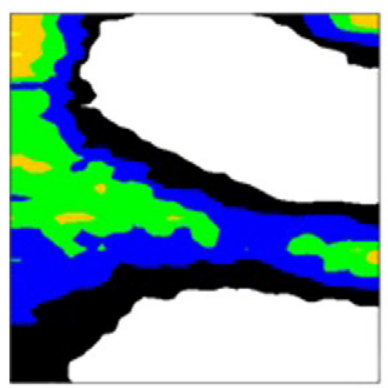

(a)

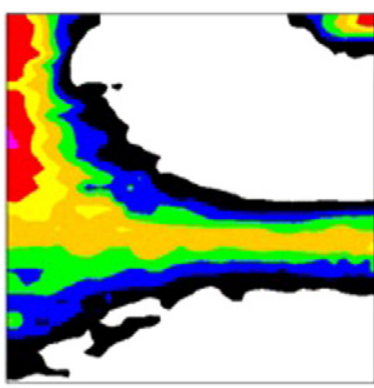

(b)

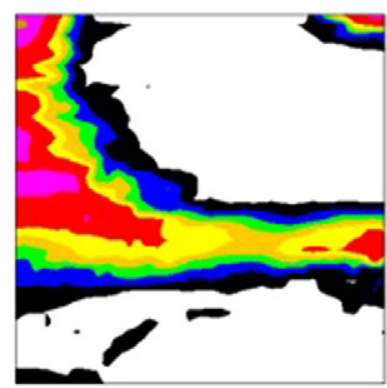

(c)

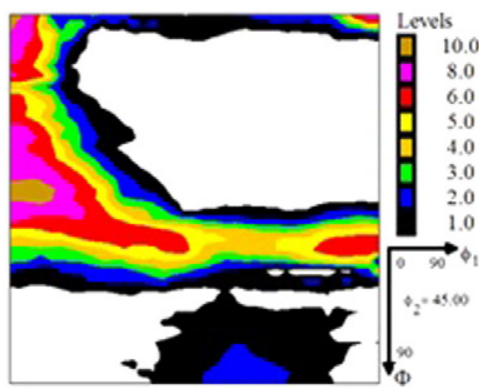

(d)

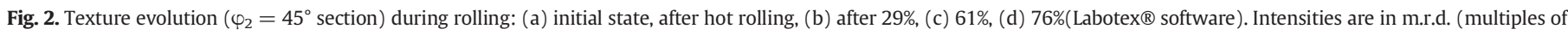
random density).

orientations which are separated by $6^{\circ}$ only). For all orientations, diffracted intensity was measured along the diffracting vector $(\theta-2 \theta$ scans) on $\{110\},\{200\},\{211\},\{220\},\{310\}$ and $\{400\}$ diffracting planes according to theoretical positions on the pole figures, which were selected as follows: first, the positions of all possible poles (namely the $\{110\},\{200\},\{211\}$ and $\{310\}$ poles) of the 6 selected orientations (including all orthotropically symmetrical orientations) were plotted. These are presented in Fig. 5. As it can be seen, the number of poles is quite large on the $\{211\}$ and $\{310\}$ pole figures, due to the multiplicity of these families of crystallographic planes. Then, for each orientation and each diffracting plane family, one pole has been selected which respects the double constraint of being as close as possible to the center of the pole figure (in order to minimize goniometer rotations and to maximize the penetrating depth of the X-ray beam) and as far as possible from the poles of the other orientations. The selected positions have been highlighted in red in Fig. 5 for the 6 orientations within one quarter of each pole figure, in which all other labels have been erased for the sake of clarity. It is seen that the selected points do avoid any overlapping of orientations for the $\{200\}$ and $\{110\}$ poles, but that it is not the case for the $C$ orientation on the $\{310\}$ pole figure (where it is very close to an $\mathrm{F}$ pole), and not the case either for the $\mathrm{B}$ (close to E) and C (close to F) orientations on the $\{211\}$ pole figure. We can thus expect a higher degree of overlapping for the $\{211\}$ measurements.

Also, since XRD only provides an average measurement along the fiber determined by the orientation of the diffraction vector, the corresponding data can additionally contain some information coming from minor texture components of the fiber (which form at least $25 \%$ of the overall texture, see Table 2 ). In order to verify that their contribution to the measured intensity is negligible, two ODFs have been calculated for each strain level, by considering either the sole 6 orientations and associated volume fractions (see Table 2 ) or all remaining orientations composing the rest and the background. Then, the corresponding pole figures have been plotted. One example of such a calculation is shown in Fig. 6 for the $29 \%$ cold rolled texture and $\{200\}$ and $\{110\}$ pole figures: it is clear that the orientations which compose the rest and the background, which represent $40 \%$ of the texture in the selected example, are quite scattered and as a

Table 2

Calculated volumes fractions of the 6 selected orientations; the spread around each orientation is set to $\pm 7.5^{\circ}$ and the background to $10 \%$.

\begin{tabular}{lccccc}
\hline & Initial & $29 \%$ & $61 \%$ & $76 \%$ & $88 \%$ \\
\hline A & 4.35 & 9.69 & 10.48 & 10.07 & 9.13 \\
B & 5.45 & 8.72 & 12.28 & 12.65 & 12.65 \\
C & 4.15 & 8.13 & 9.16 & 9.6 & 8.04 \\
F & 3.53 & 6.63 & 7.4 & 8.15 & 7.65 \\
D & 4.05 & 6.94 & 7.15 & 7.55 & 6.5 \\
E & 7.2 & 13.18 & 15.36 & 16.84 & 15.88 \\
Rest & 61.27 & 36.7 & 28.16 & 25.15 & 30.75 \\
Background & 10 & 10 & 10 & 10 & 10 \\
\hline
\end{tabular}

consequence, their contribution to the measured peaks can be considered to be negligible.

Some examples of Bragg peaks are now shown in Fig. 7: the peaks have been normalized and centered, and are presented in two different scales, in order to emphasize the main characteristics of these peaks. The normalization is rendered necessary by the fact that the counting parameters are not always the same, since they depend on the actual spread of the scanned peak as well as on the intensity of the diffracting orientation. It is worth mentioning though that the signal to noise ratio varies between $10^{3}$ in the worst case to $10^{4}$ in most of the cases, which is remarkably high and typical of synchrotron measurements. When the peaks are plotted in a logarithmic scale, it becomes obvious that the tails are entirely captured (the lowest values are very close to the background value) and that these parts present a very small scatter.

It is also worth noting that, in the present case, all measured Bragg peaks are remarkably symmetrical (see Fig. 7). This could be an indication of a low level of long-range internal stresses associated with the cell-wall dislocation structure at the level of the texture component since this is not the case when measurements are performed in single crystals [20,23-27]. However, in highly deformed grains, peak asymmetry can also be due to the polarization of the dislocation microstructure, i.e. the fact that when dislocation boundaries are formed during plastic strain, the dislocations of a certain sign can be stopped on one side of the boundary and those of opposite sign on the other side of the boundary. This polarity may give rise to an asymmetry of the slip resistance [28]. We can thus probably anticipate that, in the present case, the investigated microstructures (measured at the level of one texture

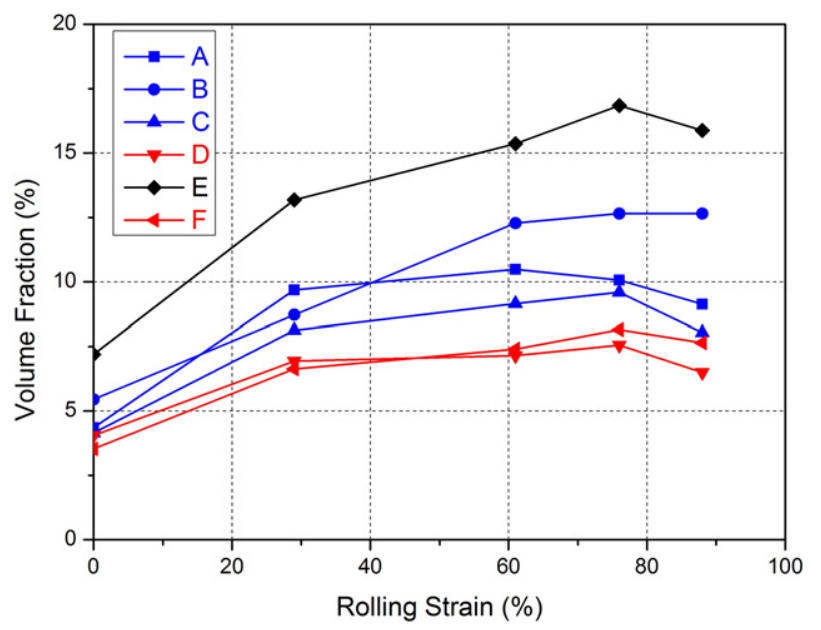

Fig. 3. Calculated volume fractions of the various texture components as a function or rolling strain. 


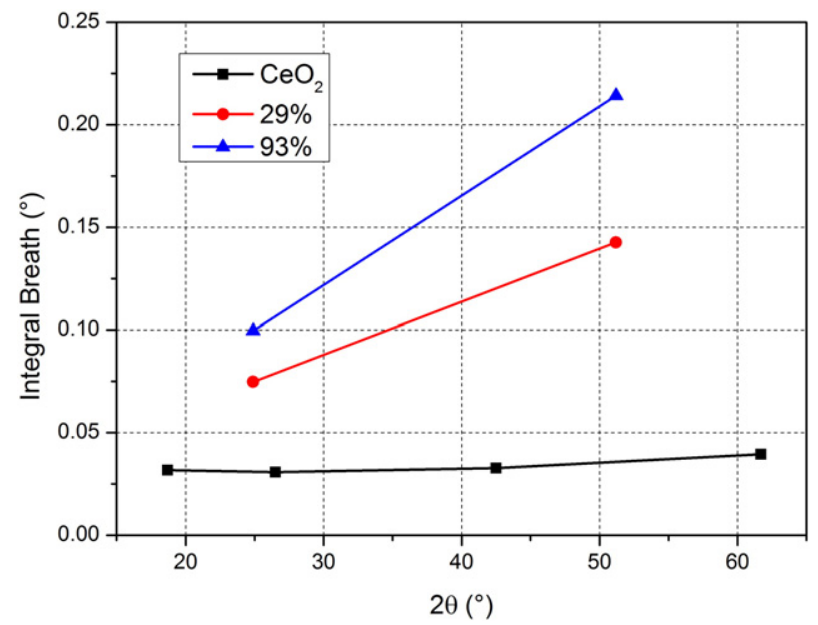

Fig. 4. Values of integral breadth for different analyzed planes for the reference powder $\mathrm{CeO}_{2}$ ( $\{220\},\{400\},\{620\}$ and $\{840\}$ planes), the less (29\% strain) and the most deformed samples (93\% strain) ( $\{200\}$ and $\{400\}$ planes). The measurements are performed for specimen orientations corresponding to the center of the pole figures, which always correspond to a high texture intensity for the deformed samples.

component) are not strongly polarized. In other words, even if the microstructure is composed of dislocation cells, these must be relatively homogeneous in size and more or less randomly oriented within the investigated volume (which is composed of several grains of the same orientation). This can be due to the fact that the fragmentation of the grains during deformation is due to the simultaneous effect of the increase of the dislocation content within the dislocation walls and of subsequent different reorientations during strain of the various scanned subgrains. In that context, we can then assume that the concept of a restrictedly random distribution of dislocations introduced by Wilkens [17] may provide an adapted model for our microstructures, and this justifies a priori the use of Eq. (3) for the stored energy, as well as the Fourier analysis presented below.

We can further argue that this conclusion has an additional consequence on the various dislocation densities present in the material (Eqs. (1a) and (1b)). Considering that our measurements correspond to several grains associated with the same orientation, the total dislocation density given by Eq. (1a) can be simplified to $\rho_{\text {tot }}=\rho_{w}+\rho_{c}$, the third term $\rho_{G B}$ - estimated to be much smaller than the other two, see e.g. $[11,29]$ - being included into the $\rho_{w}$ term as a contribution to the so-called GND density (see Introduction section). Now, two different assumptions can be made before analyzing the XRD peaks:

(i) Either these XRD measurements give access to $\rho_{c}$ only, but the absence of internal stresses implies then that $[23,24]$

$\rho_{c}=\rho_{w}$

and the measured density can then simply be multiplied by a factor of 2 in order to get the complete dislocation density $\rho_{\text {tot }}$.

(ii) Or, both cell and wall (sub-grain or grain boundaries) contribute to the measurements, again in more or less equal proportions because of the observed absence of internal stresses, and we measure directly $\rho_{\text {tot }}$ by XRD in that case.
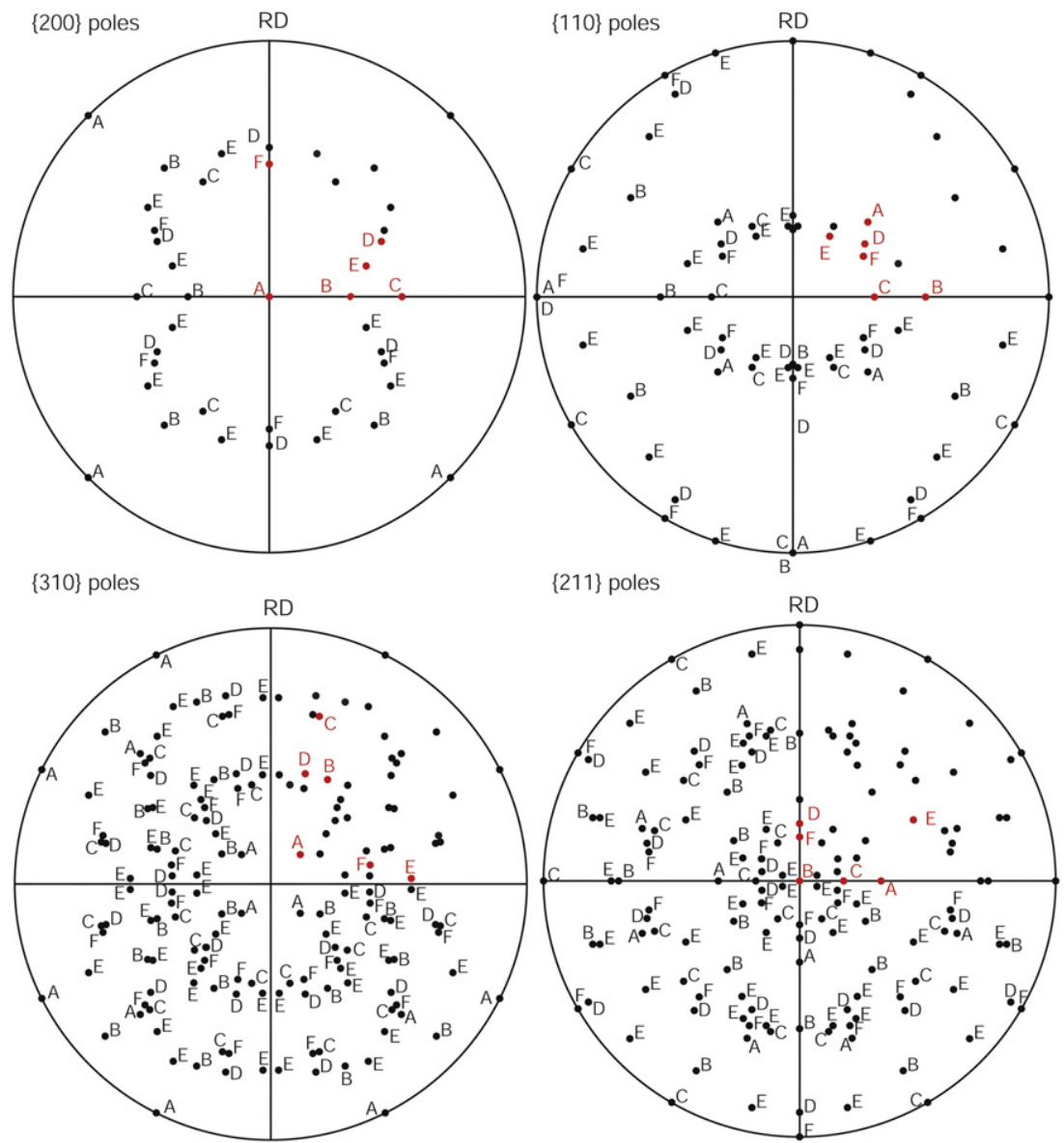

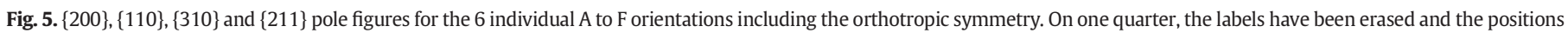
selected for high resolution X-ray diffraction have been highlighted in red. 
(a)
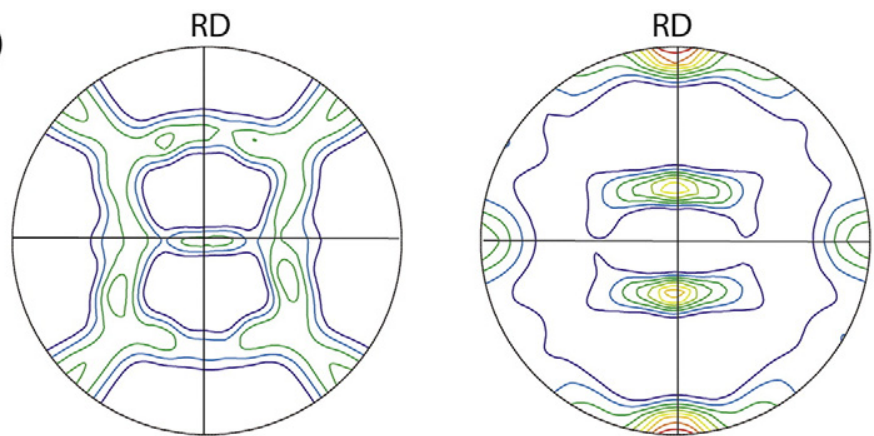

$\{200\}$ poles, $\max =2.90$

$\{110\}$ poles, $\max =5.80$

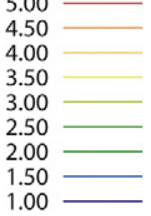

(b)

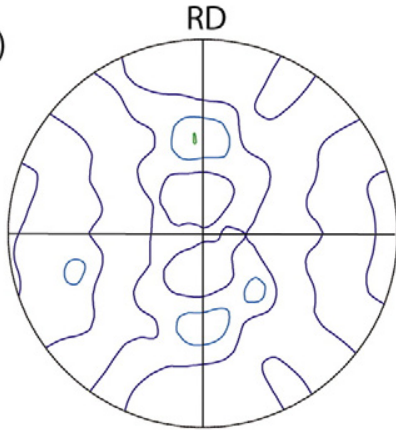

$\{200\}$ poles, $\max =1.90$

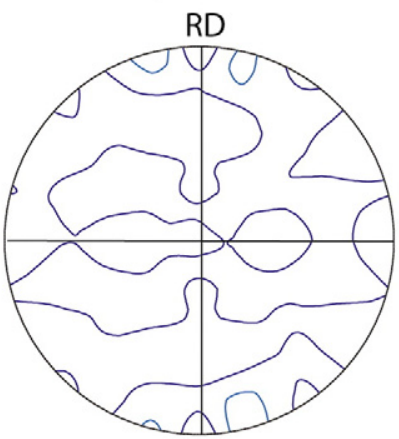

$\{110\}$ poles, $\max =5.80$

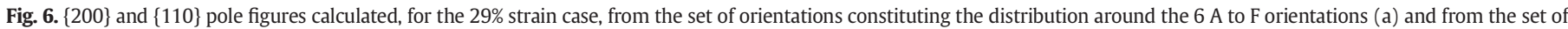
orientations constituting the remaining part of the texture and the background (b).

This must be kept in mind when comparing measured values to published ones. But in both cases, it will be possible to quantitatively compare values obtained by XRD in different orientations, as long as the relative contribution of the walls and the cells is indeed comparable. It is interesting to note that in recent papers relating measurements or calculations of dislocation densities from XRD peaks, e.g. [30-33], the question of the possible influence of the wall dislocations into the evaluation of the average dislocation density is never addressed. Although - to the authors' knowledge - this fact has never been either proved or inferred, it seems that, in all mentioned cases, it is explicitly assumed that indeed $\rho_{\text {tot }}=\rho_{w}+\rho_{c}$ is evaluated in case of symmetrical peaks, whereas $\rho_{c}$ and $\rho_{w}$ are separately evaluated in the case of asymmetrical peaks. In the present work, we will simply call the measured density $\rho_{X R D}$ and try to deduce from our analysis whether we are closer to hypothesis (i) $\rho_{X R D}=\rho_{c}$ or (ii) $\rho_{X R D}=\rho_{\text {tot }}$.

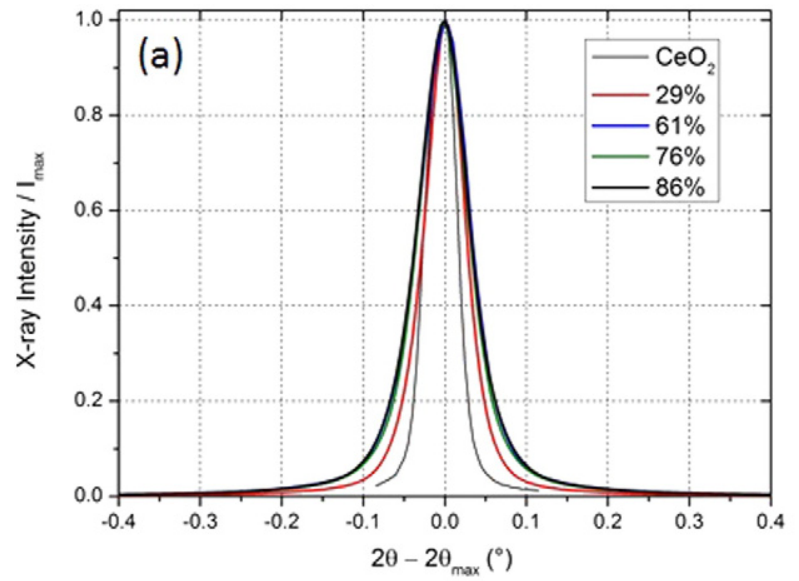

\section{Analysis method for high resolution diffraction data}

\subsection{The used line broadening model}

Our investigated material being very low alloyed and highly deformed, we first assume that both broadening and shape of the diffraction peaks may be affected only by the size of the coherent domains and the presence of dislocations. We thus can make use of the modified Warren Averbach (mWA) method introduced by Wilkens and further developed by Ungar and coworkers [24-26,33] to analyze the XRD data. The principle is the following: Bragg diffraction is sensitive to the position of atoms in the specimen. Dislocations distort the crystal lattice, creating a displacement field of atoms which signature is contained (in a statistical way) in the shape of any Bragg peak. The mWA method provides a (relatively simple) way to extract the dislocation density from

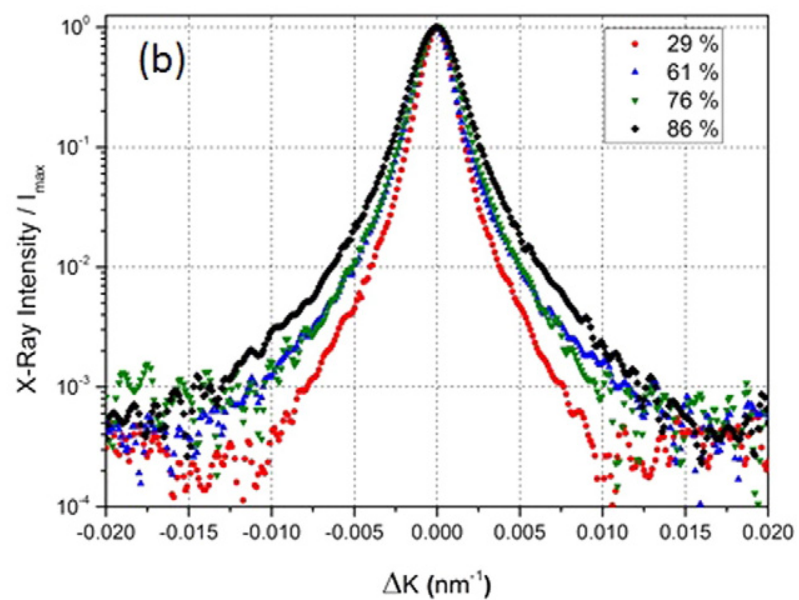

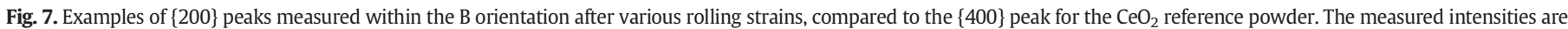
normalized by the maximum one. In Fig. $7 \mathrm{~b}, K=2 \sin \theta / \lambda$. 
the peak shape. Some other statistical parameters of the dislocation structure (for example polarization) can also be estimated using higher order moments of the dislocation peak, e.g. describing peak asymmetry. We do not go that far in this work since data treatment can become sensitive. The measured Bragg peaks $I(2 \theta)$ have to be expressed in the crystal reciprocal lattice space $I(\mathbf{K})$, with $\mathbf{K}$ being the diffraction vector. According to the mWA model, the Fourier Transform $A(L)$ of $I(\mathbf{K})$, for small values of $L$, reads

$$
\begin{aligned}
\ln |A(L)|= & \ln \left|A^{S}(L)\right|-\frac{\pi b^{2}}{2} \rho_{X R D} L^{2} \ln \left(\frac{R_{e}}{L}\right) K^{2} \bar{C} \\
& +Q b^{2} L^{4} \ln \left(\frac{R_{2}}{L}\right) \ln \left(\frac{R_{3}}{L}\right)\left(K^{4} \bar{C}^{2}\right) .
\end{aligned}
$$

Here, $L$ corresponds to a physical length in the crystal in the direction of the diffraction vector, $K$ is the norm of the diffraction vector $\mathbf{K}$ ( $K=$ $2 \sin \theta / \lambda$, with $\lambda$ the wavelength), $\rho_{X R D}$ is the dislocation density, and $R_{e}$ an outer cut-off radius for the dislocations $\left(R_{e} \approx 1 / \sqrt{\rho_{X R D}}\right)$. In the last term of Eq. (6), $Q, R_{2}$, and $R_{3}$ are additional material parameters. This Fourier transform is indeed linked to the various moments of the atomic displacement field within the investigated volume. This formula has been obtained by doing some assumption on the dislocation structure, e.g. all dislocation segments are considered to be straight.

The first term $A^{s}$ expresses the broadening due to the small size of the diffracting subgrains, and which becomes important essentially for sizes smaller than $\sim 100 \mathrm{~nm}$. The dimensionless coefficient $\bar{C}$ is the average contrast factor of the dislocations present within the material, which accounts for the anisotropy of the deformation field created by the dislocations [34]. This factor can be calculated, as long as some hypotheses are made about the number of slip systems populated by dislocations and the possible dislocation types present in the material (see below).

\subsection{The influence of the number of considered slip systems}

In the literature, the following standard assumptions can be found:

(i) It is often considered that all possible slip systems are equally populated and that it is not possible to really distinguish between edge and screw type; in that case, half of the dislocations are considered of edge type and the other half are screws. This approximation is often shown to be consistent with the peak broadening analysis [30,35,36];

(ii) Crystal plasticity is sometimes used to reduce the number of populated systems; this approach is most often shown to improve $[11,16,37]$ or more rarely to deteriorate the quality of the fitting procedure (exposed below) [38]; it is sometimes completed by somewhat arbitrary assumptions on the dislocation character [11];

(iii) All possible slip systems are taken into account and assumed to be equally populated, but the character (screw, edge or mixed) is not a priori set, but deduced from a minimization process [31].

Ideally, it seems tempting to use a polycrystalline model to get a more precise idea on the actual number of active systems and to deduce the dislocation character from the peak analysis. However, since most of the models, used up to now, do not take explicitly into account the building up and the recovery of dislocation microstructures, there is no reason why the number of predicted active systems should reflect the number of experimentally observed populated systems. Another alternative consists in using a DDD (discrete dislocation dynamics) code to evaluate the number of populated systems and type of dislocations. This has been done recently to assess the validity of the Wilkens model [32]. However, if this procedure is convenient for small strains in single crystals, it cannot be applied anymore to the case of strongly deformed polycrystals, due to the tremendous effects of stress and strain localization associated with the mechanical interaction between neighbor grains. To our opinion, taking thus all systems equally populated (as in point (i) above) seems to be closer to the reality in this case, since it integrates the total deformation history and associated changes of active systems.

\subsection{The influence of the dislocation character}

As for the dislocation character, it is not at all easy to identify it experimentally (see e.g. [39]). In that case, the adjustment procedure seems tempting. But this does not seem appropriate in the case of bcc metals, in which the screw dislocations are known to glide much slower than the edge ones [39-41]. As a consequence, mainly screw types remain in the material after deformation. We are thus going to make use of this observation and assume for simplification that all dislocations present in our material are of screw character. During the Fourier analysis, this assumption will also be compared to the classical half screw/half edge assumption. The corresponding average (on all systems) contrast factors are listed in Table 3 for the two different hypotheses ( $50 \%$ screw $+50 \%$ edge or $100 \%$ screw). Note that if the values of the $K^{2} \bar{C}$ coefficients are not so different between the two cases, the points corresponding to the various diffracting planes will not be placed in the same order in the plot of the Fourier coefficients as a function of $K^{2} \bar{C}$ (Eq. (6)) and this will of course strongly affect the quality of the fit. An example of such a fit by Eq. (6) of the Fourier coefficients of the peaks measured in the F orientation after 76\% strain is shown in Fig. 8 , assuming that all dislocations are screw. The plotted curves are obtained by a least square fit on the coefficients obtained on various peaks and for various $L$ values, through a homemade software. This procedure allows the determination of all material parameters composing Eq. (6). The very good quality of the fit justifies a posteriori the assumption made on dislocation character and renders useless any additional minimization procedure, which would most probably lead to a very similar result, i.e. a stronger screw character (see Section 3.2).

Also, in this example as in all other treated cases, imposing $50 \%$ screw and $50 \%$ edge dislocations leads to a much poorer fit of the experimental data by Eq. (6) (not shown here). It is also worth mentioning though that two peaks, namely the $\{211\}$ and $\{400\}$, have been systematically taken out from the present analysis. Indeed, for the $\{211\}$ measurements, it has been seen previously that it was difficult in the present case to get data corresponding solely to one main texture component. As for the $\{400\}$ measurements, due to the much larger value of $K^{2} \bar{C}$ (see Table 3 ), the quality of the adjustment is slightly reduced when those peaks are taken into account.

\section{Presentation and analysis of the experimental results}

\subsection{Assessment of the XRD dislocation density}

The results of the X-ray analysis described in Section 3 by considering only screw dislocations are now presented in Table 4 and Fig. 9. The evolution of the dislocation density is quite different for the $\alpha$ and $\gamma$ fiber orientations. In the first deformation steps, the dislocation density increases mainly in the $\alpha$ orientations (at least up to 20\% for the B

Table 3

Calculated averaged contrast factors for all investigated planes with the 2 selected hypotheses.

\begin{tabular}{cllrcrrr}
\hline & & $\{110\}$ & $\{200\}$ & \multicolumn{1}{c}{211} & \multicolumn{1}{c}{220} & \multicolumn{1}{c}{310} & \multicolumn{1}{c}{400} \\
\hline 1/2 Edge 1/2 & $\bar{C}$ & 0.1414 & 0.2736 & 0.1416 & 0.1414 & 0.2260 & 0.2736 \\
Screw & $K^{2} \bar{C}$ & 3.4421 & 13.3199 & 10.3405 & 13.7685 & 27.5086 & 53.2798 \\
100\% Screw & $\bar{C}$ & 0.104 & 0.3055 & 0.104 & 0.104 & 0.2329 & 0.3055 \\
& $K^{2} \bar{C}$ & 2.5317 & 14.8730 & 7.5966 & 10.1268 & 28.3485 & 59.4919
\end{tabular}




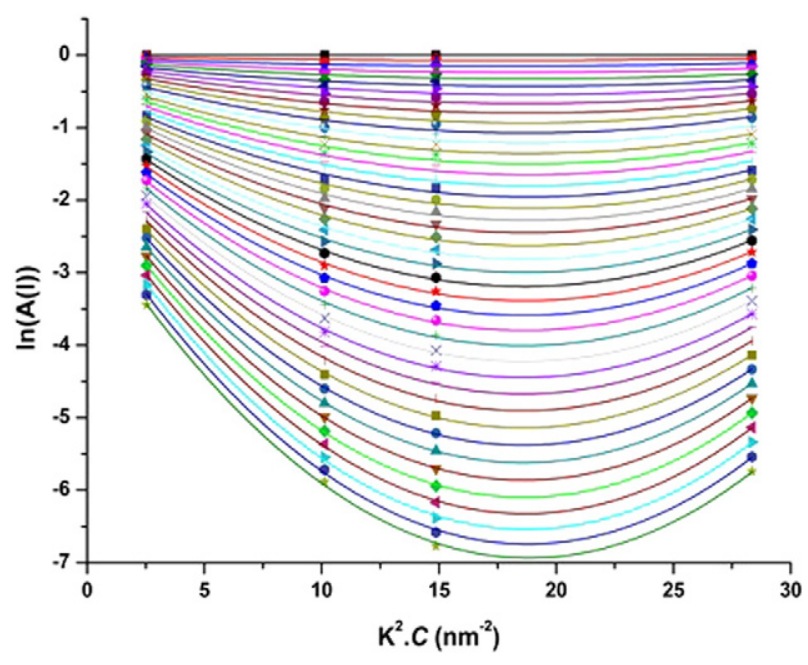

Fig. 8. Application of the mWA method on a specimen cold-rolled to $76 \%$ for the F orientation $(\{111\}<112>)$. Here, Eq. (6) nicely matches the Fourier transform of $\{110\},\{220\}$, $\{200\}$ and $\{310\}$ planes, up to $\mathrm{L}=66.3 \mathrm{~nm}$ (in this figure, $\mathrm{L}$ decreases from the top $(\mathrm{L}=0 \mathrm{~nm})$ to the bottom $(\mathrm{L}=66.3 \mathrm{~nm})$ with a step of $\Delta \mathrm{L}=1.7 \mathrm{~nm})$.

component but possibly up to a larger strain like e.g. around $40 \%$, and up to $60 \%$ for the $A$ and $C$ orientations), while it is more or less constant in the other orientations. After $60 \%$ strain, the dislocation density increases strongly in the F orientation while it decreases strongly in all $\alpha$ orientations. Additionally, it is seen that the dislocation density is more or less constant in the D and $\mathrm{E}$ orientations which are considered as orientations intermediate between $\alpha$ and $\gamma$ fibers. At this stage, we can also say that the present data are only partly consistent with previous ones, as mentioned in Section 1: in the present work, the observed hierarchy between various texture components is clearly dependent on the strain level, and possibly strongly affected by the initial texture.

As these orientations become more or less stabilized with strain, it is tempting to conclude at this stage that the observed different evolutions are the consequence of different hardening and softening mechanisms which can take place within the different orientations during deformation; these mechanisms are not only dependent on the number and nature of active slip systems, but also on the degree of reorientation which will affect in turn the fragmentation of the grains [42], and thus the various dislocation densities listed in Section 2.

\subsection{Assessment of the reorientation capacity of the various orientations}

In order to visualize the possible degree of reorientation of each fragmented grain, a simple polycrystalline model can be used to calculate the reorientation field for each point of the Euler space. This has been done here with a viscoplastic Taylor model, which assumes that all grains of a polycrystalline sample are subjected to the same imposed strain rate tensor, and which takes into account $12\{110\}<111>$ and 12 $\{112\}<111>$ slip systems with the same reference shear stress [43]. Then, the Euler space (here, only the $\varphi_{2}=45^{\circ}$ section) is discretized into regularly spaced orientations, and one single plane strain compression step is imposed to each of them. The assessment of the slip rate on all active slip systems enables then to calculate the three independent

Table 4

Measured dislocation densities $\left(\mathrm{m}^{-2}\right)$ using Eq. (6), considering only screw dislocations.

\begin{tabular}{lllll}
\hline Orientation & $29 \%$ & $61 \%$ & $76 \%$ & $88 \%$ \\
\hline A & $2.59 \mathrm{E}+15$ & $7.75 \mathrm{E}+15$ & $4.10 \mathrm{E}+15$ & $3.67 \mathrm{E}+15$ \\
B & $6.38 \mathrm{E}+15$ & $3.81 \mathrm{E}+15$ & $1.35 \mathrm{E}+15$ & $1.56 \mathrm{E}+15$ \\
C & $3.18 \mathrm{E}+15$ & $6.99 \mathrm{E}+15$ & $4.28 \mathrm{E}+15$ & $1.91 \mathrm{E}+15$ \\
F & $3.73 \mathrm{E}+15$ & $2.44 \mathrm{E}+15$ & $7.25 \mathrm{E}+15$ & $6.70 \mathrm{E}+15$ \\
D & $3.33 \mathrm{E}+15$ & $2.94 \mathrm{E}+15$ & $2.88 \mathrm{E}+15$ & $2.49 \mathrm{E}+15$ \\
E & $2.48 \mathrm{E}+15$ & $2.52 \mathrm{E}+15$ & $2.24 \mathrm{E}+15$ & $2.16 \mathrm{E}+15$ \\
\hline
\end{tabular}

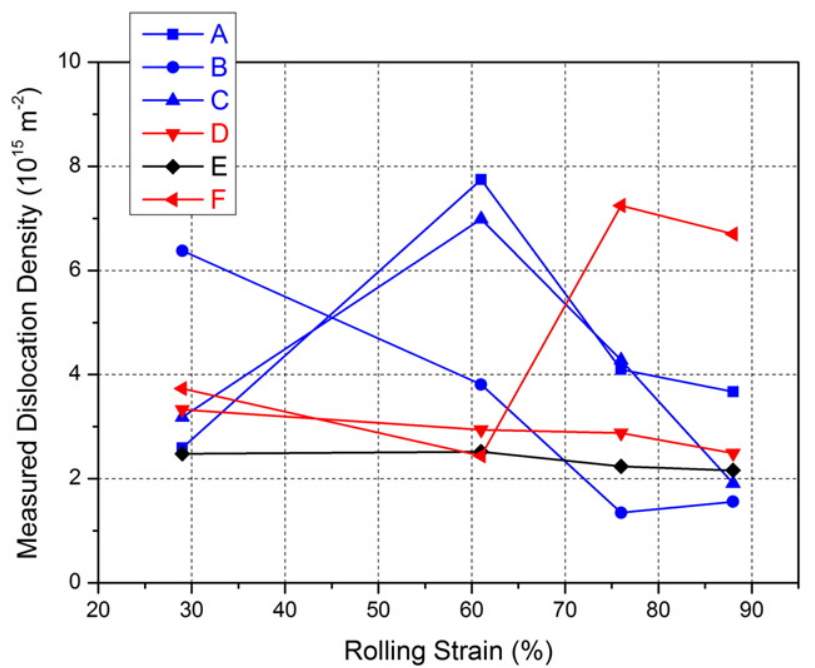

Fig. 9. Dislocation densities extracted from the peak analysis.

components of the lattice spin tensor $\Omega_{23}, \Omega_{13}$, and $\Omega_{12}$. The components of orientation change can then be deduced from the following evolution laws for the 3 Euler angles [44]:

$\dot{\varphi}_{1}=-\Omega_{12}-\dot{\varphi}_{2} \cos \Phi$

$\dot{\Phi}=-\Omega_{23} \cos \varphi_{1}-\Omega_{31} \sin \varphi_{1}$

$\dot{\varphi}_{2}=-\Omega_{23} \frac{\sin \varphi_{1}}{\sin \phi}+\Omega_{31} \frac{\cos \varphi_{1}}{\sin \phi}$.

From these equations, the three increments $\Delta \varphi_{1}, \Delta \Phi, \Delta \varphi_{2}$ can then be easily deduced. In Fig. 10, each considered orientation is represented by a dot whose size is proportional to $\Delta \varphi_{2}$ (related to reorientation outside the $\varphi_{2}=45^{\circ}$ section), and associated with a small line whose components are proportional to $\Delta \varphi_{1}$ and $\Delta \Phi$, and which represents reorientation inside the $\varphi_{2}=45^{\circ}$ section. It is interesting to note that this figure confirms the fact that the orientations A and B are stable, whereas a general reorientation of orientations from $F$ to $B$ through

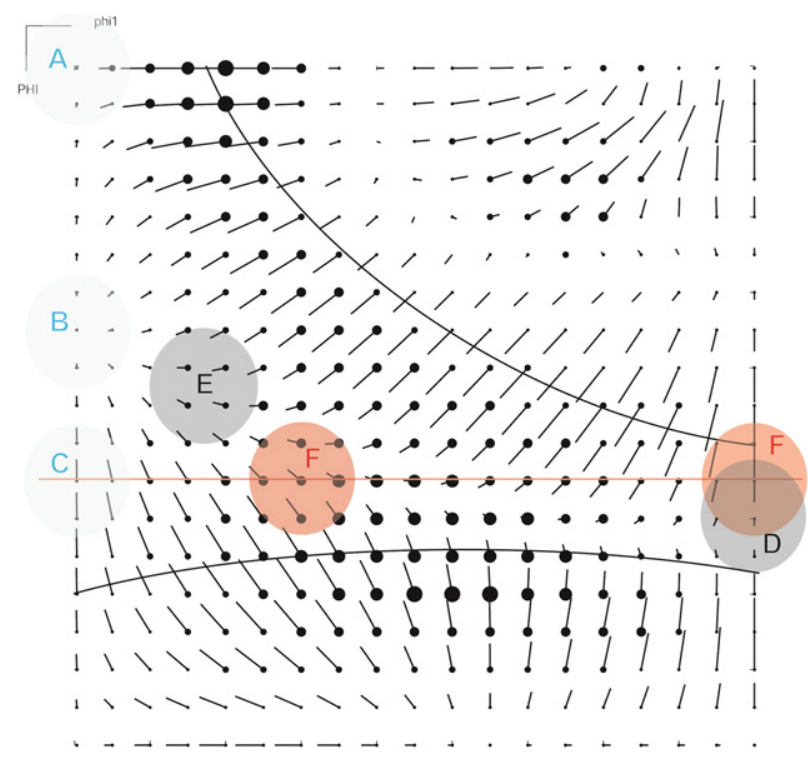

Fig. 10. Reorientation map calculated with the Taylor model. The large circles represent the spread around each studied orientation used for percentage calculations (see Table 2). The two black lines delimitate the zone occupied by the initial texture of the material (see Fig. 2). 
E is predicted by such a model (with a main rotation around TD). The $C$ orientation tends also to reorient towards $B$ through a rotation around RD. The area occupied on this section by the initial texture (see Fig. 2) is also identified in this figure (by the two extreme lines), which enables to understand why the E orientation remains highly populated up to large strains while the B orientation is reinforced. It is also worth noting that as soon as a dislocation substructure is built up inside a given grain, if the associated orientation is unstable, the various subgrains are generally subjected to slightly different reorientation rates, and this can further enhance the grain fragmentation and modify the dislocation density within the subgrain boundaries [42].

\subsection{Assessment of the fragmentation from the EBSD measurements}

This fragmentation can easily be visualized by EBSD. Some microstructures measured on the RD-ND plane are shown in Fig. 11. It is clear that some fragmentation takes place in some grains at the very early stage of deformation, but that it is difficult to distinguish different behaviors in $\alpha$ and $\gamma$ grains, especially at large strains.

In order to go further, some microstructural parameters have been extracted from these maps. The first one is the average intercept along the RD or the ND. This intercept is defined here as the distance along a line parallel to RD or ND between two boundaries associated with misorientations larger than $10^{\circ}$ or $1^{\circ}$. This parameter allows estimating the grain size even when the material is highly deformed. It can then be calculated for a given map along several lines parallel to RD or ND in such a way to have at least one measurement per grain, and then simply averaged. These different intercepts are represented in Fig. 12 for the two selected limiting misorientation values. As the misorientation limit of $10^{\circ}$ is quite close to the generally accepted limit between grain boundaries and subgrain boundaries, it is obvious that the observed evolution is indeed associated with grain fragmentation (since, without fragmentation, the grain size and thus the intercept value should constantly increase along $\mathrm{RD}$, as a result of plane strain compression; this is indeed the case below $15 \%$ strain). It is also clear that there is a transition between two different regimes between $30 \%$ and $50 \%$ strain: fragmentation gradually increases within the grains up to $40 \%$ strain where it reaches the limiting value of $10^{\circ}$ and then gradually saturates, especially after $60 \%$ strain; this could be due to the combined effect of fragmentation and elongation of the grains together with the recovery of the subgrain and grain boundaries (which will directly affect the density of GNDs). As for the intercept calculated with the misorientation value of $1^{\circ}$, which directly characterize the subgrain boundaries or dislocation cells, a similar observation of a general saturation after $60 \%$ can also be drawn.

To further quantify this subgrain boundary dislocation density, one second parameter can be extracted from the EBSD map, i.e. the local intragranular misorientation inside grains belonging to specific texture components (fiber or individual orientation). It is calculated here as the so-called kernel average misorientation (KAM), i.e. the average misorientation between a point on the measurement grid and its nearest

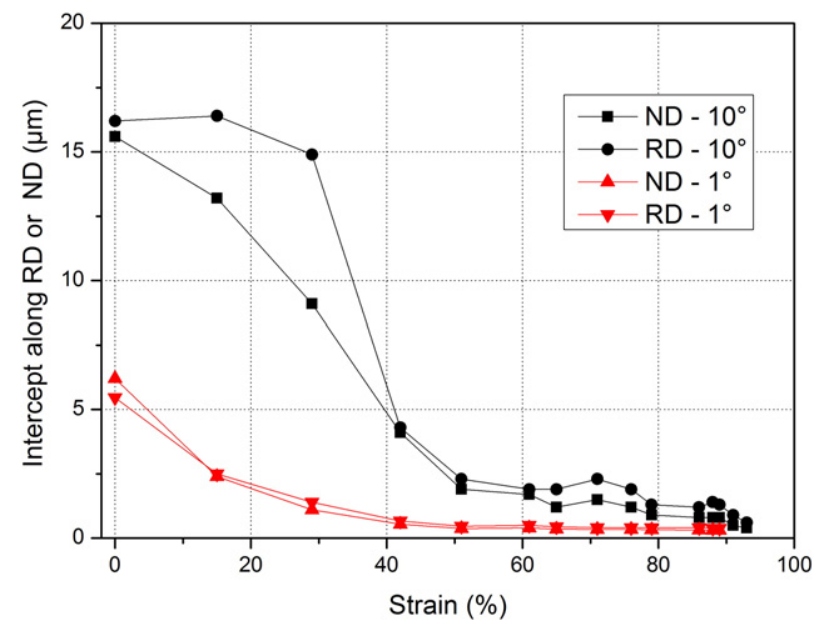

Fig. 12. Evolution of the intercept along RD or ND with strain, for two different misorientation values $\left(10^{\circ}\right.$ or $\left.1^{\circ}\right)$

neighbors. To ensure that all considered points belong to the same grain, misorientations larger than $5^{\circ}$ have been excluded from this average. For one given texture component, a frequency histogram can then be plotted, and the peak value, i.e. the misorientation value associated with the maximum of the histogram can then be extracted. This parameter, characterizing the intragranular misorientation, is plotted in Fig. 13 as a function of strain for $\alpha$ and $\gamma$ grains. Again, a transition between two regimes is visible around 60\%: intragranular misorientations increase first slowly similarly in all grains, whereas it increases more significantly in the $\gamma$ grains than in the $\alpha$ grains after $60 \%$.

\subsection{What happens during rolling?}

It is worth noting first that when we measure a diffraction peak within a given orientation during strain, we are in a way in an Eulerian scheme: we focus on one orientation but not on specific grains. In other words, we examine here three different types of grains: (i) some grains associated with stable orientations from the beginning of the deformation, (ii) some grains which are unstable at the beginning of the strain path and which will always remain unstable because the strain level is not high enough or (iii) some unstable grains which will finally reach some stable orientations. Thus, the observed behavior of one orientation is indeed the collective behavior of various grains, whose initial orientation was not necessarily the same [44]. Also, if some papers relate the hardening behavior of single crystals (e.g. [45]), the hardening/softening behavior of specific orientations within a polycrystalline sample is rarely documented in the literature, and mostly associated with moderate strains only [46]. Furthermore, the previous modeling efforts clearly demonstrate that the results are indeed strongly dependent on the selected polycrystalline model $[47,48]$.

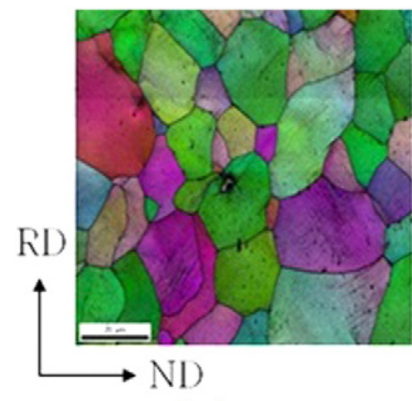

(a)

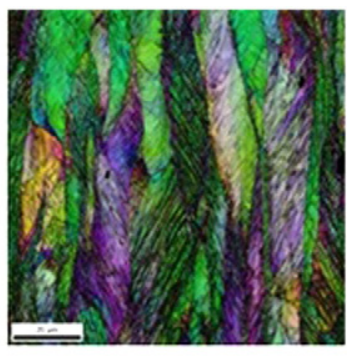

(b)

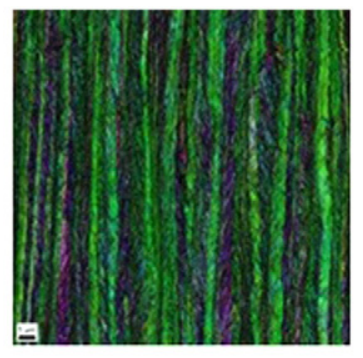

(c)

Fig. 11. EBSD maps measured on the transverse section of the cold-rolled material after (a) $15 \%$, (b) $61 \%$ and (c) $88 \%$ rolling strains. Maps of $100 \mu \mathrm{m} \times 100 \mu \mathrm{m}$, step size of $0.2 \mu \mathrm{m}$. 


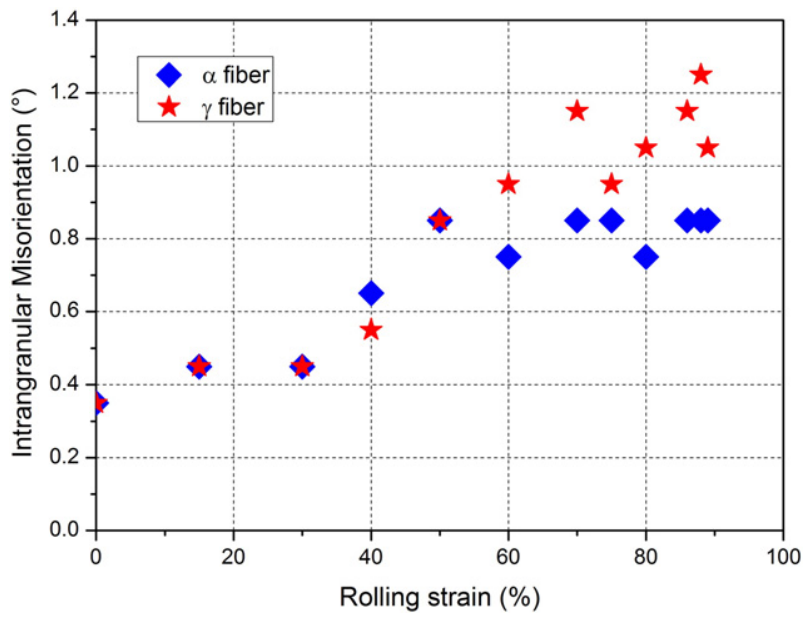

Fig. 13. Evolution of the intragranular misorientation measured by EBSD (calculated as the misorientation value at the peak of the Kernel histogram for EBSD maps of $100 \mu \mathrm{m}$ by $100 \mu \mathrm{m}$ measured with a step size of $0.2 \mu \mathrm{m}$ ) in $\alpha$ and $\gamma$ fiber grains as a function of strain.

Having said that, can we still try to explain the observed evolutions in terms of GND and SSD densities? It seems difficult at this stage to separate the two, although some experimental facts are worth recalling. Indeed, it was already qualitatively observed that, during complex strain paths, these two dislocation populations did have different evolutions within different orientations [44]: both $\rho_{c}$ and $\rho_{w}$ were observed to (i) first increase and then decrease with strain within stable orientations (first $\rho_{c}$ and then $\rho_{w}$ ), (ii) remain quite low within unstable orientations and (iii) to increase again in stabilized and fragmented orientations.

Based on these previous observations and on the simultaneous inspection of both X-ray and EBSD data as well as Taylor calculations, we can then propose the following scenario for the microstructural evolution taking place in various grains during rolling:

From 0 to $40 \%$ strain. All grains harden and slightly fragment (see Fig. 13), but the dislocation density $\rho_{X R D}$ increases mainly in the $\alpha$ fiber, and especially in the stable A and B orientations. Those, as well as the $E$ orientation are strongly reinforced because of the texture evolution (see Fig. 8). But as the A and B orientations are stable, the fragmentation of these grains is only due to the building up of a dislocation substructure (both $\rho_{c}$ and $\rho_{w}$ increase significantly, as in [44]) whereas fragmentation is enhanced by (or even mostly due to) reorientation in the E orientation and thus presumably associated with a lower $\rho_{w}$ dislocation density since reorientation favors the absorption of dislocations within cell walls (it is also the case in the C, F and D orientations, which are all unstable at this stage). We can thus conclude at this point, that both cell and wall dislocations present in orientations $\mathrm{A}$ and $\mathrm{B}$ are indeed taken into account in our X-ray analysis (otherwise, $\rho_{X R D}$ would not increase so significantly in $\mathrm{A}$ and $\mathrm{B}$, compared to the other orientations).

From 40 to $60 \%$ strain. The same scenario continues, but the level of dislocation density has now reached a saturation value in $\mathrm{B}$; as a result, recovery of the microstructure takes place within this orientation provoking a strong decrease of both $\rho_{c}$ and $\rho_{w}$. The dislocation density increases mostly in A but also in the $\mathrm{C}$ orientation, whose fragmentation due to reorientation is now reduced (the overall texture intensity increases slightly).

From 60 to $80 \%$ strain. The saturation dislocation density value is reached in $\mathrm{A}$ and $\mathrm{C}$, and thus recovery of the microstructure takes also place in these orientations, leading to a strong decrease of the overall dislocation density $\rho_{X R D}$ together with a stagnation of the intragranular misorientation. Because of texture evolution, the spread of orientation becomes now reduced around F (see Fig. 2), and we observe an enhancement of intragranular fragmentation in this orientation (see also Fig. 13), associated with a strong increase of the dislocation density $\rho_{X R D}$. These observations are similar to those performed in the B orientation at low strains, and we can thus conclude that both $\rho_{c}$ and $\rho_{w}$ increase significantly at this stage in the F orientation, leading in turn to a strong increase of $\rho_{X R D}$. After $80 \%$ strain. Only the stable B orientation is still reinforced, and the overall reorientation towards this final orientation is slower and slower. Since the saturation value has also been reached in the $\mathrm{F}$ orientation, recovery becomes active in this orientation leading to a slight decrease of the dislocation density.

Also, we can notice that the ratio of $\rho_{X R D}\{222\} / \rho_{X R D}\{200\}$ (or more precisely $\rho_{X R D}(\mathrm{~F}) / \rho_{\text {XRD }}(\mathrm{A})$ in the present investigation) evolves here from about 0.5 after $50 \%$ strain to about 2.0 after $90 \%$ strain (unlike in the previously reported analyses, where this ratio was always claimed to be larger than 1 , see Section 1 ). The initial hot rolling texture, mainly composed of components close to the $\alpha$ fiber, and its subsequent evolution during cold rolling could be largely responsible for this result as proposed above.

If the observed evolution of the dislocation density can be quite surprising, especially the fact that we observe strong decrease of $\rho_{X R D}$ within some orientations for various levels of strain, it is nevertheless consistent with previous studies: for example, based on the scenario initially proposed by Ridha and Hutchinson [49] reporting an enhanced recovery within Cube oriented grains deformed in plane strain compression for copper, a hardening/softening law was recently proposed [48] which allows to account for this mechanism, and which is completely consistent with prior X-ray $\rho_{X R D}$ measurements [50]. More recently, a strong decrease of the dislocation density has also been observed in various fcc metals subjected to severe plastic strain at room temperature $[51,52]$.

It is finally worth noting that if the value of the outer cut-off radius $R_{e}$, extracted from the peak analysis also evolves with strain, the evolution of the stored energy due to the dislocations (Eq. (3)) is exactly the same as the one of the dislocation density shown in Fig. 9. If we recall that, during annealing, nucleation of recrystallization always starts in the areas associated with maximum stored energy (or equivalently dislocation density) (see e.g. [53]), we can thus conclude that the present data are completely consistent with the general observation of the initiation of recrystallization of steels within the $\alpha$ fiber after moderate strains and within the $\gamma$ fiber after larger strains (see e.g. [54]), even though the actual nucleation mechanisms may be radically different in both cases (e.g. strain induced boundary migration at low strains versus intergranular nucleation at higher strains).

\section{Conclusions}

In this work, dislocation densities have been estimated from X-ray diffraction measurements performed in several texture components of a rolling IF steel sheet, after several strain levels, using synchrotron radiation. The proposed analysis is based on the Fourier analysis of the peaks, by considering that all possible slip systems are equally populated but that all remaining dislocations are of screw character.

The main findings of our work are the following:

- The measured peaks have been shown to be highly symmetrical for all investigated orientations; to our opinion, this implies that the scanned dislocation microstructures are not polarized, which could suggest that the measurements are statistically representative of orthotropic texture components;

- The assumption of only screw dislocations has been shown to provide a very good fit for all investigated texture components; 
- The evolution of the dislocation density varies differently with strain from one texture component to another; it increases in the $\alpha$ fiber during the first deformation steps, before increasing within the $\gamma$ fiber; this can be partly explained by simultaneously looking at the texture evolution;

- With the aid of additional EBSD observations and simple calculations, it is proposed that the measured dislocation densities do include the contribution of both cells and walls (SSD and GND).

In order to go further, these measurements should be repeated in a material presenting a stronger texture, enabling to differentiate between various components without any ambiguity.

\section{Acknowledgments}

The authors would like to thank Nathalie Boudet for her help during experiment (BM02) and acknowledge the ESRF for beamtime allocation.

\section{References}

[1] J. Humphreys, M. Hatherly, Recrystallization and Related Annealing Phenomena, Second editionElsevier, Oxford, 2004.

[2] P. Gerber, J. Tarasiuk, T. Chauveau, B. Bacroix, A quantitative analysis of the evolution of texture and stored energy during annealing of cold rolled copper, Acta Mater. 51 (20) (2003) 6359-6371.

[3] B. Bacroix, O. Castelnau, A. Miroux, R.H., Relations between deformed grain orientations, stored energy and nucleation characteristics, 21st RISO International Symposium on Materials Science, 2000 (Roskilde, Denmark)

[4] X. Huang, J.J. D., N. Hansen, Effect of grain orientation on deformation structure and recrystallization behavior of tensile strained copper, 4th International Conference on Recrystallization and Related Phenomena, JIM, Tsukuba City, Japan, 1999.

[5] A. Samet-Meziou, A.L. Etter, T. Baudin, R. Penelle, Relation between the deformation sub-structure after rolling or tension and the recrystallization mechanisms of an IF steel, Mater. Sci. Eng. A 473 (1-2) (2008) 342-354.

[6] N. Hansen, X. Huang, G. Winther, Grain orientation, deformation microstructure and flow stress, Mater. Sci. Eng. A 494 (1-2) (2008) 61-67.

[7] C. Chen, M.P. Wang, S. Wang, Y.L. Jia, R.S. Lei, F.Z. Xia, B. Zuo, H.C. Yu, The evolution of cold-rolled deformation microstructure of $\{001\} 110$ grains in Ta-7.5 wt\%W alloy foils, J. Alloys Compd. 513 (2012) 208-212.

[8] J. Jiang, T.B. Britton, A.J. Wilkinson, Measurement of geometrically necessary dislocation density with high resolution electron backscatter diffraction: effects of detector binning and step size, Ultramicroscopy 125 (2013) 1-9.

[9] A. Wauthier, H. Réglé, R. Brenner, Evolution of the deformed substructure obtained by cold-rolling in IF steel, in: P.B. Prangnell, P.S. Bate (Eds.),Fundamentals of Deformation and Annealing 2007, pp. 205-210.

[10] A. Wauthier, Observation expérimentale et prévision des paramètres de la déformation déterminants pour la genèse des textures cristallographiques lors de la recristallisation des tôles minces d'acier à base de teneur en carbone( $\mathrm{PhD}$ Thesis) Université Paris 13, 2008.

[11] A. Borbély, J.H. Driver, T. Ungár, An X-ray method for the determination of stored energies in texture components of deformed metals; application to cold worked ultra high purity iron, Acta Mater. 48 (8) (2000) 2005-2016.

[12] L.P. Evers, W.A.M. Brekelmans, M.G.D. Geers, Non-local crystal plasticity model with intrinsic SSD and GND effects, J. Mech. Phys. Solids 52 (10) (2004) 2379-2401.

[13] M.F. Ashby, Deformation of plastically non-homogeneous materials, Philos. Mag. 21 (170) (1970) 399-

[14] R.L. Every, M. Hatherly, Annealing of low-carbon drawing grade steels, J. Australas Inst. Met. 19 (3) (1974) 186-193.

[15] T. Ungar, O. Castelnau, G. Ribarik, M. Drakopoulos, J.L. Bechade, T. Chauveau, A. Snigirev, I. Snigireva, C. Schroer, B. Bacroix, Grain to grain slip activity in plastically deformed $\mathrm{Zr}$ determined by X-ray micro-diffraction line profile analysis, Acta Mater. 55 (3) (2007) 1117-1127.

[16] A. Borbély, T. Ungár, X-ray line profiles analysis of plastically deformed metals, C. R. Phys. 13 (3) (2012) 293-306.

[17] M. Wilkens, Quantitative interpretation of X-ray line broadening of plastically deformed crystals, J. Appl. Crystallogr. 8 (1975) 191-192.

[18] A. Samet-Meziou, A.L. Etter, T. Baudin, R. Penelle, Stored energy characterization after low deformation by rolling or tension of an IF-Ti, in: S.J.L. Kang, et al., (Eds.)Recrystallization and Grain Growth III, Pts 1 and 2 2007, pp. 323-328.

[19] M. Wilkens, in: S.J. A., d.I. R., B. R. (Eds.), Fundamental Aspects of Dislocation Theory, Vol. II, vol. 317, Natl Bur. Stand. (US) Spec. Publ., Washington, 1970.

[20] O. Castelnau, M. Drakopoulos, C. Schroer, I. Snigireva, A. Snigirev, T. Ungar, Dislocation density analysis in single grains of steel by X-ray scanning microdiffraction, Nucl. Inst. Methods Phys. Res. A (467-468) (2001) 1245-1248 (Part 2).

[21] N. Rajmohan, Y. Hayakawa, J.A. Szpunar, J.H. Root, The determination of orientationdependent stored energy using neutron diffraction, Phys. B Condens. Matter 241-243 (1998) 1225-1227.
[22] K. Pawlik, Determination of the orientation distribution function from pole figures in arbitrarily defined cells, Phys. Stat. Sol. B 134 (1986) 477

[23] A. Borbély, G. Hoffmann, E. Aernoudt, T. Ungár, Dislocation arrangement and residual long-range internal stresses in copper single crystals at large deformations, Acta Mater. 45 (1) (1997) 89-98.

[24] I. Groma, T. Ungar, M. Wilkens, Asymmetric X-ray line broadening of plastically deformed crystals. 1. Theory, J. Appl. Crystallogr. 21 (1988) 47-53.

[25] T. Ungar, A. Borbely, The effect of dislocation contrast on X-ray line broadening: a new approach to line profile analysis, Appl. Phys. Lett. 69 (21) (1996) 3173-3175.

[26] T. Ungar, J. Gubicza, G. Ribarik, A. Borbely, Crystallite size distribution and dislocation structure determined by diffraction profile analysis: principles and practical application to cubic and hexagonal crystals, J. Appl. Crystallogr. 34 (2001) 298-310.

[27] I. Groma, D. Tüzes, P.D. Ispánovity, Asymmetric X-ray line broadening caused by dislocation polarization induced by external load, Scr. Mater. 68 (2013) 755-758.

[28] B. Peeters, S.R. Kalidindi, C. Teodosiu, P. Van Houtte, E. Aernoudt A theoretical investigation of the influence of dislocation sheets on evolution of yield surfaces in singlephase B.C.C. polycrystals, J. Mech. Phys. Solids 50 (2002) 783-807.

[29] D. Mandal, I. Baker, Measurement of the energy of grain boundary geometricallynecessary dislocations in copper, Scr. Metall. Mater. 33 (1995) 831-836.

[30] R.A. Renzetti, H.R.Z. Sandim, R.E. Bolmaro, P.A. Suzuki, A. Möslang, X-ray evaluation of dislocation density in ODS-Eurofer steel, Mater. Sci. Eng. A 534 (2012) 142-146.

[31] T. Shintani, Y Murata, Evaluation of the dislocation density and dislocation character in cold rolled Type 304 steel determined by profile analysis of X-ray diffraction, Acta Mater. 59 (11) (2011) 4314-4322.

[32] L. Balogh, L. Capolungo, C.N. Tomé, On the measure of dislocation densities from diffraction line profiles: a comparison with discrete dislocation methods, Acta Mater. 60 (4) (2012) 1467-1477.

[33] T. Ungár, Dislocation densities, arrangements and character from X-ray diffraction experiments, Mater. Sci. Eng. A 309-310 (2001) 14-22.

[34] T. Ungár, I. Dragomir, A. Révész, A. Borbély, The contrast factors of dislocations in cubic crystals: the dislocation model of strain anisotropy in practice, J. Appl. Crystallogr. 32 (1999) 992-1002.

[35] G. Mohamed, S. Bacroix, J.L. Lebrun, Anisotropy of dislocation distribution and longrange internal stresses, in: A.J. Bottger, R. Delhez, E.J. Mittemeijer (Eds.),Ecrs 5: Proceedings of the Fifth European Conference on Residual Stresses 2000, pp. 285-290.

[36] J. Pešička, R. Kužel, A. Dronhofer, G. Eggeler, The evolution of dislocation density during heat treatment and creep of tempered martensite ferritic steels, Acta Mater. 51 (16) (2003) 4847-4862.

[37] G. Guiglionda, A. Borbély, J.H. Driver, Orientation-dependent stored energies in hot deformed $\mathrm{Al}-2.5 \% \mathrm{Mg}$ and their influence on recrystallization, Acta Mater. 52 (12) (2004) 3413-3423.

[38] G. Mohamed, B. Bacroix, T. Ungar, J.L. Raphanel, T. Chauveau, Experimental and numerical determination of the intragranular work hardening in a cold rolled multicrystal, Mater. Sci. Eng. A 234 (1997) 940-943.

[39] B.W. Lagow, I.M. Robertson, M. Jouiad, D.H. Lassila, T.C. Lee, H.K. Birnbaum, Observation of dislocation dynamics in the electron microscope, Mater. Sci. Eng. A 309-310 (2001) 445-450.

[40] D. Caillard, Kinetics of dislocations in pure Fe. Part I. In situ straining experiments at room temperature, Acta Mater. 58 (9) (2010) 3493-3503.

[41] S. Naamane, G. Monnet, B. Devincre, Low temperature deformation in iron studied with dislocation dynamics simulations, Int. J. Plast. 26 (2010) 84-92.

[42] J.Y. Kang, B. Bacroix, H. Regle, K.H. Oh, H.C. Lee, Effect of deformation mode and grain orientation on misorientation development in a body-centered cubic steel, Acta Mater. 55 (15) (2007) 4935-4946.

[43] B. Bacroix, Z.Q. Hu, Texture evolution during strain path changes in low carbon steel sheets, Metall. Mater. Trans. A Phys. Metall. Mater. Sci. 26 (3) (1995) 601-613.

[44] E.V. Nesterova, B. Bacroix, C. Teodosiu, Microstructure and texture evolution under strain-path changes in low-carbon interstitial-free steel, Metall. Mater. Trans. A Phys. Metall. Mater. Sci. 32 (10) (2001) 2527-2538.

[45] A.S. Keh, Work hardening and deformation sub-structure in iron single crystals deformed in tension at $298^{\circ} \mathrm{K}$, Philos. Mag. 12 (1965) 9-30.

[46] C.C. Merriman, D.P. Field, P. Trivedi, Orientation dependence of dislocation structure evolution during cold rolling of aluminum, Mater. Sci. Eng. A 494 (2008) 28-35.

[47] B. Bacroix, A. Miroux, O. Castelnau, Simulation of the orientation dependence of stored energy during rolling deformation of low carbon steels, Model. Simul. Mater. Sci. Eng. 7 (1999) 851-864.

[48] B. Bacroix, R. Brenner, A phenomenological anisotropic description for dislocation storage and recovery processes in fcc crystals, Comput. Mater. Sci. 54 (2012) 97-100.

[49] A.A. Ridha, W.B. Hutchinson, Recrystallisation mechanisms and the origin of cube texture in copper, Acta Metall. 30 (10) (1982) 1929-1939.

[50] P. Gerber, J. Tarasiuk, T. Chauveau, B. Bacroix, A quantitative analysis of the evolution of texture and stored energy during annealing of cold rolled copper, Acta Mater. 51 (20) (2003) 6359-6371.

[51] Y. Murata, I. Nakaya, M. Morinaga, Assessment of strain energy by measuring dislocation density in copper and aluminium prepared by ECAP and ARB, Mater. Trans. 49 (1) (2008) 20-23.

[52] W. Wei, K.X. Wei, G.J. Fan, A new constitutive equation for strain hardening and softening of fcc metals during severe plastic deformation, Acta Mater. 56 (17) (2008) 4771-4779.

[53] G. Mohamed, B. Bacroix, Role of stored energy in static recrystallization of cold rolled copper single and multicrystals, Acta Mater. 48 (13) (2000) 3295-3302.

[54] W.B. Hutchinson, Development and control of annealing textures in low-carbon steels, Int. Metall. Rev. 29 (1984) 25-42. 